\title{
The Optimal Design and Study of the Power Ultrasonic Transducer Vibrating System
}

\author{
Xinxin Xie \\ Xi'an Aeronautical Polytechnic Institute, Xi'an 710089, Shaanxi, China
}

Keywords: Power ultrasonic transducer vibrating system; optimal design; research.

\begin{abstract}
With the continuous development of the economy, the development of our country's science and technology has entered a new phase. Subsequently, the power ultrasound technology has been optimized, among which the vibrating system has played an important role, mainly including the ultrasonic transducer, the ultrasonic amplitude transformer, the ultrasonic tool head and the ultrasonic radiator. This essay is aimed at the analysis and research of the optimal design and study of the power ultrasonic transducer vibrating system.
\end{abstract}

\section{Introduction}

The previous vibrating system of high power ultrasonic transducer is mainly composed of high power ultrasonic transducer, the ultrasonic amplitude transformer and the ultrasonic tool head, of which the rational allocation and optimal design have a direct effect on the vibration performance, mainly including the design of the power ultrasonic transducer, the ultrasonic amplitude transformer and the ultrasonic tool head. Also, the optimal fusion of the three is affected. In recent years, the further study of the issue has been strengthened by the relevant personnel, contributing to many excellent results. Actually, the application of the high power ultrasonic is a further development process of the ultrasonic radiance and intensity of the system.

\section{The Optimal Design of the Ultrasonic Amplitude Transformer Engineering}

\subsection{Function of the Optimal Design of the Ultrasonic Amplitude Transformer Engineering}

The ultrasonic amplitude transformer is also called the ultrasonic energy collecting device, the ultrasonic transmission and ultrasonic solid horn. For the ultrasonic technology, the function of the ultrasonic amplitude transformer is very crucial. It is the solid ultrasonic device of high and powerful vibration amplitude that is often applied into the power ultrasonic devices and medical ultrasonic devices for treatment. The function of the ultrasonic amplitude transformer in the power ultrasonic transducer vibrating system is just like that of the click transformer in the electronic devices, mainly as followed. Firstly, the transformation effect of mechanical vibration mainly includes changes between the vibration displacement, the vibration velocity and the stress. Secondly, the impedance of matching effect is aimed at the exchange of mechanical impedance between the energy transducer and the mechanical loading, for the match between energy transducers in accordance with the sound. Thirdly is the fixed effect, to fix the system and reduce energy consumption by the application of flange plate into the displacement of the amplitude lever. Fourthly, in case of some extreme ultrasonic environment, such as the high temperature and strong corrosion environment, the ultrasonic amplitude transformer could act as the buffer unit to protect the ultrasonic energy transducer.

\subsection{Choice of Materials}

Since the rational selection of the material of ultrasonic amplitude has a direct effect on the performance of the vibrating system, it will generate higher mechanical carrying tension and energy consumption if the material used is in high-frequency vibration. In addition, the uniformity and hardness are also required to interconnect with the energy transducer and coupling facility, which generates very small radiation and contributes to the improvement of processing and chemical stability. The ultrasonic amplitude transformer, used during the dealing process by the ultrasound, often chooses tool steel, titanium alloy, brass and so on as its materials. When in real application, to 
ensure the accuracy of design, the material density and the propagation velocity of the modulus of elasticity and elastic wave should be in overall grasp.

\section{Optimal Design of Tool Head for Power Ultrasonic Vibration System}

\subsection{Function of the Optimal Design of Tool Head on Power Ultrasonic Vibration System}

With respect to the energy transducer design of the power piezoelectric ceramics, what is mainly concerned by the relevant personnel is the effect of the pre-stress on choosing, controlling, measuring and the performance of the energy transducer. At the present stage, among the already formed power ultrasonic and underwater acoustic transducers, there are three main ways to apply pre-stress, like the central single-helix method, outer circumferential poly-helix and the external stressed sleeve. Considering the uniform and portability of the pre-stress, now the construction of many energy transducers is the application of the central single crew.

The function of transducer in pre-stressing is mainly reflected in four aspects. Firstly, within the specified frequency range, pre-stress can adjust the resonance rate of transducer. Secondly, reasonable pre-stressing of transducer can promote the effective contact between transducer and areas, promoting the improvement of electric-acoustic efficiency and power capacity of transducer. Thirdly, too small or too large longitudinal pre-stress cannot be carried out. If the pre-stress is relatively small, the prestress will have an impact on the performance of piezoelectric ceramic materials, but if the pre-stress is too large, the vibration of the transducer will be restrained and the piezoelectric ceramic element will be damaged. In addition, the mechanical stress will reduce the coupling coefficient of the transducer. Fourthly, if more piezoelectric ceramic materials is used, the pre-stress should be about $250 \mathrm{~kg} / \mathrm{cm}$.

\subsection{Electromechanical Coupling Coefficient of Transducer Rationality}

One of the most important factors in the optimization design of transducer is the effective electromechanical coupling coefficient of transducer, which is directly related with transducer efficiency and bandwidth. The effective electromechanical coupling coefficient of the transducer will become larger and larger, if the piezoelectric ceramic element exists around the displacement node of the transducer. The total length of the piezoelectric ceramic element in the transducer needs to be suitable, with the optimal length being $1 / 4$ to $1 / 3$, in order to ensure the coupling coefficient of the larger effective electro mechanics. If the impedance matching material is used, the coupling coefficient of the effective electromechanical of the transducer is to be improved.

\subsection{The Impact Analysis of the Interface of the Transducer Components in the Performance of} the Transducer.

The smoothness and roughness of the interface in all components of the transducer have unprecedented influences in the performance of the transducer. First, the contact surface should be smooth, ensuring the effective propagation of sound waves; Second, we must control the roughness of the interface itself, to ensure the area of effective contact area and further promote contact surface smoothness and flatness of ascension. It can also reduce the mechanical loss between interfaces and promote the efficiency of electroacoustic transducer. The measures adopted are mainly to improve the machining accuracy. In a strict occasion, it is necessary to grind the contact surface, and at the same time, grind the surface of the piezoelectric ceramic element; in addition, if the central screw is used to hold a fixed sandwich type piezoelectric ceramic transducer, it will cause a certain change in the surrounding metal towards stressing. If the closed contact degree between the transducer interfaces will not make a difference due to the contact deformation in metal plate, then we need to reprocess before and after the metal cover plate, which will help to compensate for the deformation of the bolt. In this way, the design of power ultrasonic vibration system can be optimized. 


\section{New Progress in Optimization Design of Power Ultrasonic Vibration System.}

\subsection{Analysis of Ultrasonic Vibration System of Radiation Power in All Directions Under the Vibration Mode.}

The vibration mode converted to a full range of radiation power ultrasonic vibration system belongs to a kind of radiation device of three-dimensional ultrasonic wave. The main features include three aspects. Firstly, the utilization of metal radiation cylinder before and after both ends of the transducer realizes the transducer radiates back and forth at the same time, overcomes the single surface radiation of ultrasonic transducers under conventional power and tremendously improves the radiation efficiency of transducer; Secondly, influenced by the concept of Poisson effect and coupled Vibration of Materials, we use the radial and longitudinal vibration coupling in the metal circular tube to realize the two-dimensional radial radiation of the transducer, and effectively improve the radiation efficiency of the transducer. Thirdly, the design of transducer size is made by reasonable methods. The diameter and height of the metal circular tube itself, for example, can change to the coupling between transducer longitudinal and radial vibration of the vibration, thus realizing the vertical vibration system of metal circular tube and radial vibration strong resonance at the same time, to enlarge the range of the radiation power and ultrasonic role. Fourthly, the radiation area of the vibration system increases gradually, improves the system matching, and then improves the vibration system electroacoustic conversion rate Thus, using the structure innovation of the vibration system could realize three-dimensional radiation vibration system of ultrasonic power, and process technology in the liquid of high power ultrasonic, such as the ultrasonic wave of the extraction and ultrasonic oil extraction and ultrasonic cleaning as well as the biological technology for a wide range of applications.

The purpose of the vibration system in the design is mainly to produce ultrasonic threedimensional radiation, so the radial dimension of the system is especially large. The front and rear metal radiation cylindrical and intermediate metal tube in the system both vibrate the threedimensional coupling, so the vibration in the whole system is very complicated and cannot be resolved. Influenced by the condition, it can use finite element method of numerical simulation and the simulation of system as a whole, and also constantly optimize the system vibration modal and the displacement distribution and the radiation sound field characteristics, which could be used in ultrasonic occasions, a different power. Only in this way can the power ultrasonic vibration system be optimized and designed.

\subsection{Ultrasonic Transducer Analysis of Radial Sandwich Piezoelectric Ceramics}

Radial sandwich piezoelectric ceramic composite ultrasonic transducer has several main advantages in the optimization design. First, the area of the transducer radiation is large, so it is convenient to match the sound, so it can produce high power radiation. Second, the acoustic radiation belongs to two dimensions, which can promote the increase of the range of sound waves. Third, it is convenient for the acoustic radiation in the pipeline, through the choice of different metal materials; it can promote the realization of acoustic radiation both inside and outside the sound wave. At this stage, the transducer is just at the stage of laboratory development and performance simulation, and theoretical analysis and simulation of transducer and basic experimental test have been completed, but there are still a series of technology problems. What is more important is the transducer radial pre-stress application and the problems of control, and there are some radial pre-stress applying methods which mainly have heat bilges cold shrink and bonding method with pre-stressed method of casing. Among them, heat bilges cold shrink method is chosen more frequently. However, this method has very strict requirements for part of the transducer geometry size. When it cannot be effectively controlled, it will be likely to lead to the radial pre-stress being too big or too small. If the pre-stressed is too large, it will make piezoelectric ceramic pipe fracture, and if too small, it can cause bad contact for transducer, and then increase the mechanical loss of the transducer and mechanical quality factor decreases gradually. Although the adhesive method is very simple, the power transducer cannot be used because of the binding strength of adhesive. In addition, pre-stressing is simple, but it needs to be noted that if grooves are used in the outer metal tube of the transducer, this will affect the integrity of the transducer structure and the uniformity of radial radiation and the mechanical strength 
of the transducer and the high-power performance. If it is a sandwich type piezoelectric ceramic transducer with high power radial composition, and the transducer itself has a large size, then the piezoelectric elements in the transducer are required to have the specified geometrical dimensions. Affected by the existing process conditions, a single large size of piezoelectric ceramic pipe is difficult to process, and it also cannot ensure the product consistency between the geometry size and performance, so a variety of short piezoelectric circular tube axial superposition can be chosen.

In addition, other new types of high-power piezoelectric ultrasonic transducer vibration system have been applied in China at the same time, for example, using a single transducer excitation on the tube end, or using two transducers on both ends of the tube excitation, respectively. In order to improve the effect of ultrasonic treatment, the exploration of complex frequency transducers should be strengthened in related researches, and the effective analysis and comparison between different design schemes and technologies need also be made. As for the Spanish scholar's high-power gas medium ultrasonic transducer, the characteristics of the transducer is very unique, and is able to be specifically applied in the special environment and technology. In addition, among such technologies of ultrasonic motor and ultrasonic machining, compound vibration modes of the ultrasonic transducer such as longitudinal-torsional composite and composite buckling mode ultrasonic transducer have obtained better development.

\section{Conclusion}

In summary, the testing technology of power ultrasonic transducer is closely related with the development of ultrasonic transducer. And the former is usually applied to the performance realization of high-powered ultrasonic transducer, for example, ultrasonic power and ultrasonic cavitation field. The optimization of vibration system is connected with the development of the age. And electronic technology, automation control technology as well as machinery manufacturing are the significant factors to cause effect on the constant optimization of ultrasonic transducer. The current development direction mainly includes the high conversion rate, low price and without environment pollution, and the increasingly improving transducer mechanism after the optimization and fabrication processing of transducer.

\section{References}

[1]. Yang Zhang. The device design of Ultrasonic-pressure coupling and its effect on the microstructure of Al-Cu alloy[D]. South China University of Technology, 2016.

[2]. Xiaojun Xian. Research on the Transducer of 1-3-2 Piezoelectric Composite Material[D]. Shaanxi Normal University, 2016.

[3]. Lelin Zhang. Parametric Design of Power Ultrasonic Honing Device under the Environment of NX[D]. North University of China, 2016.

[4]. Hua Tian. Research on the New-type Ultrasonic Transducer and Radiator[D]. Shaanxi Normal University, 2016.

[5]. Hongjie Zhang. Design and Control of Conversion System with High Frequency for Hot Ultrasonic Bonding[D]. Tianjin University, 2012.

[6]. Long $\mathrm{Xu}$. Design and Optimization of Power Ultrasonic Vibration System with the Mode of Conversion[D]. Shaanxi Normal University, 2011. 